\title{
Sensing the Audience in Digital Streaming: Lessons from a Global Pandemic
}

\author{
ANA LEVORDASHKA \\ University of Bath, Bath, UK, a.levordashka@bath.ac.uk \\ DANAË STANTON FRASER \\ University of Bath, Bath, UK, d.stantonfraser@bath.ac.uk \\ IAIN D. GILCHRIST \\ University of Bristol, Bristol, UK, i.d.gilchrist@bristol.ac.uk \\ PAUL HILL \\ University of Bristol, Bristol, UK, paul.hill@bristol.ac.uk \\ ELEANOR CHADWICK \\ Sleight of Hand, Bristol, UK, ellie_chadwick@icloud.com
}

Live performances are immersive shared experiences, traditionally taking place in designated, carefully designed physical spaces such as theatres or concert halls. As it is becoming increasingly common for audiences to experience this type of content remotely using digital technology, it is crucial to reflect on the design of digital experiences and the technology used to deliver them. This research is guided by the question: How can the design of streaming technologies support artists in creating immersive and engaging audience experiences? A series of audience studies, which took place as cultural organisations were forced to adapt and deliver their content remotely due to the COVID19 global pandemic, highlighted problems with existing streaming solutions and informed a set of design recommendations for audience experience and research.

\section{CCS CONCEPTS • Performing Arts • Design • Psychology}

Additional Keywords and Phrases: Immersive Technology, Presence, Audience Studies, Design Guidelines

\section{INTRODUCTION}

Making physical social gatherings and shared experiences virtually impossible, the global COVID19 pandemic challenged the very idea of a live audience. Despite facing financial uncertainty, the arts sector did not stand stilltechnology was utilised in creative ways and culture was very quickly brought to people's homes. This unlikely global experiment in digital delivery of performance arts led to valuable insights into how the design of technology can better support emotional experiences and social connection.

We present a set of audience studies conducted within Bristol and Bath Creative R\&D, a UK Arts and Humanities Research Council -funded Cluster of universities and creative industries. Shortly before the start of the pandemic, the Cluster initiated a programme of work around an Expanded Performance theme including a cohort of established and 
emerging creatives, exploring the challenges and opportunities around liveness and togetherness in theatre, live music and technology [ 3$]$. The audience studies reported here are early-stage pilots within this theme. The set of design recommendations that emerged from this work are valuable and extremely timely given the ongoing restrictions imposed by the pandemic. The paper is structured as follows: We provide a brief overview of key stakeholder needs based on prior literature and how they are met in existing approaches, both in-person and digital. Next, we present our own work and insights. These are brought together into a series of design recommendations that highlight challenges and opportunities for digital content delivery.

Audience experience, or how audiences perceive the value and quality of the performing arts and other media, is influenced by various factors including sensory immersion, shared experiences or "togetherness", sense-making and reflection. Sensory and cognitive immersion, often described as a "transportation" into a fictional world, is a major driver of media enjoyment and persuasiveness [7]. Experiences are particularly powerful when shared. Audiences seek and appreciate collective engagement, a sense of togetherness with performers and other audience members [15]. Also shared among many audience members is a desire to reflect, make sense of an experience, and provide feedback [15]. Audiences' intrinsic motivation to reflect on their experiences is mirrored by the need of researchers and other stakeholders to gather such feedback. Interestingly, however, the two are often decoupled and participation in audience research is low and its orchestration - costly. In discussing how to improve audience experiences, it is important to note that there are stark individual differences in audiences' preferences-immersion, togetherness, and reflection are not universally sought after and appreciated. These individual differences are important to consider when designing experiences and technology.

The physical locations in which arts are traditionally experienced, provide opportunities for the key audience needs of immersion, togetherness, and reflection, in a way that is not readily met by their digital counterparts. Physical venues are often specifically designed to enhance immersion-emphasis is placed on content, distractions are reduced or discouraged. It is even possible that stepping into a designated space, as one would do in a theatre, may produce changes in cognition, such as interrupting pre-existing cognitive processes (e.g., thoughts) and reducing the corresponding cognitive load. This idea echoes psychological research on spatial shifts, according to which walking through rooms can disrupt ongoing cognitive processes [16]. In contrast, the creators of digital content have little control over or even information about the environment of their audiences-screen size, image quality, background noise and clutter can all vary greatly. In physical spaces, togetherness - the physical co-presence of others - is felt constantly and immediately. Audiences can and do communicate in subtle ways, such as synchronized coughs, movements, applause [24]. Communication and reflection naturally take place in the liminal spaces before and after performances in lobbies, queues, restaurants, which are known to influence the overarching audience experiences [ 4 , 15]. The design of digital experiences and the technology used to support them, need to creatively incorporate these elements.

Viewing technology and digital experiences as purely subpar to reality is of course limiting-traditional physical settings do not provide perfect answers to audiences' needs, while the possibilities of technology extend beyond the mere emulation of reality. The research and design recommendations discussed in this paper are guided by the question of how technology can support audiences' needs for immersion, togetherness, and reflection. How these are accomplished in traditional settings is as an important point of information but not an ultimate gold standard.

\section{CURRENT APPROACHES TO DIGITAL CONTENT DELIVERY}

The most common ways to deliver content remotely include web players, such as YouTube or the BBC iPlayer, and video conferencing platforms, such as Zoom. Novel, innovating solutions, including platforms like Hopin [26] and BBC R\&D's Makerbox tool StoryFormer [2] are starting to emerge in response to needs not met by pre-existing tools. Audience research typically relies on post-experience questionnaires or focus groups, or in-depth laboratory studies using specialized software and equipment (e.g., $[\underline{23}, \underline{25}])$. We briefly review these existing approaches in relation to audience experience and research.

Web players provide some, but often limited, interactivity and behavioural analytics. Media content is embedded within a web page and, unless viewed in full screen, it appears alongside information, such as comments, branding and advertisement, recommendations. The amount of visual information displayed alongside media content is usually 
abundant (e.g., three quarters of users' screen in YouTube [27]). In terms of analytics, web players can provide rich data on viewing behaviour, such as who is watching and for how long, who pauses or drops out and when. Available to end users, however are only summary statistics.

Live and interactive performances can be delivered via video conferencing platforms, which allow for synchronous audio-video communication between performers and audiences. If enabled, audience visibility and interactions are direct and highly visible-audience members appear in full-definition along with their surrounding environment. This results in loss of privacy and high visual load. Users have limited control over how content and participants are displayed. Display options are selected by users, rather than event host, leaving performers and producers with no direct control over what users actually see and experience.

New tools have emerged. Hopin is a platform specifically designed for live events, which evokes a strong sense of place and co-presence. Audience interactions are possible and encouraged before and after content viewing, but interactivity during viewing is limited. Behavioural analytics and audience research functionality are not currently implemented. None of the tools reviewed so far enables interactive storytelling-a gap filled by BBC's StoryFormer. This open-source software is in early-development stage and does not include social features and analytics.

\section{AUDIENCE STUDIES}

We present three pilot studies, conducted within the Bristol and Bath Creative R\&D Cluster-an interdisciplinary collaboration between universities and the creative industry, revolving around the use of immersive technology in the arts. The studies are ongoing work with separate aims but all involved a discussion around the potential and shortcomings of existing technology to deliver performance arts to remote audiences. In addition to primary data and analyses, the insights we report come from the process of conducting these studies, the desk-based research carried out in preparation, and discussions with creative practitioners. Our design recommendations are therefore subject to further validation and aimed to start a discussion, rather than provide conclusive answers.

\subsection{Bristol Arts Channel during national Lockdown}

We worked with the organisers of the Bristol Arts Channel-an arts \& culture initiative, which took place in Bristol, UK in May 2020, at the start of a nation-wide lockdown due to the COVID19 pandemic. The aim of the initiative was to reignite a sense of community and belonging in times of drastic isolation, as well as to increase arts participation and support local artists and venues [10].

An audience survey was carried out to collect insights into audience behaviour and experiences. The survey was distributed during the final week of the programme, via the social media channels of participating organisations. It included open-ended questions on perception of the initiative, along with closed-ended measures of overall experience (willingness to recommend, also known as promoter score; 10-point scale: $1=$ Not at all likely;10=Extremely likely), emotional experiences during events (immersion, togetherness, sense of place; 10 -point scale: 1=Not at all;10=All of the time), engagement, event attendance and, demographics. Sixty-six participants took part in the survey, of which 29 completed most items. Of the respondents who chose to provide demographic information, the majority were female (23 female; 9 male,1 non-binary) and based in Bristol (51). There was a broad range of age groups, with a majority between 35-49 (17) and 50-64 (13). We further analysed the platforms on which event streaming and audience interactions took place.

Over 100 events were featured on the Bristol Arts Channel. The majority were delivered via commercial web players (YouTube, Vimeo, Facebook) and conferencing platforms (Zoom); further interactions took place via social media (Twitter, Facebook). According to our pilot survey, audiences' overall evaluation was most strongly associated with experiencing a sense of place ("Being in a "place", having a sense that the performances and audiences are local or nearby"; Pearson's correlation $\mathrm{r}(29)=.46, \mathrm{p}=.009)$, as compared to immersion $(\mathrm{r}(29)=.31, \mathrm{p}=.086)$ and togetherness $(\mathrm{r}(29)=.31, \mathrm{p}=.091)$. Experiencing a sense of place was positively correlated with togetherness $(\mathrm{r}(37)=.66, \mathrm{p}<.001)$ and immersion $(r(37)=.40, p=.012)$, but immersion and togetherness were not associated $(\mathrm{p}=0.387)$. Such pattern could be due to our observations that immersive (full-screen, low perceptual load) and social (comments, videos) are often at a trade off in commercially available streaming solutions. 
With regard to research participation, we noted that despite general interest and positive responses, participation rate was considerably low. This was not unique to our survey but a commonly faced problem. Considering the importance of audience research and evaluation for event producers and funders, improving the efficacy of audience research would be highly beneficial.

\subsection{Immersive Audio Sessions}

A research study was conducted with project partners at Real World recording studios. The primary aim of the research is to develop indirect measures of audience engagement and gather feedback on an immersive audio concert produced by Real World. The audio concert was broadcasted publicly as part of Womad at Home-a programme of online events put together for audiences of the world music festival Womad, postponed due to the COVID19 pandemic.

The audience study consisted of "Listening Sessions" held via the video conferencing platform Zoom. We tested 20 participants over 4 sessions (5-10 participants per session). Participants were asked to use their web cameras throughout the session. They were able to see each other at the beginning and end of the session, but were instructed to hide the videos from view for the duration of the concert. As a measure of momentary engagement, some participants were asked to report instances of mind-wandering by typing a pre-defined response as a private message to the session moderator. Participants were instructed to focus primarily on the concert and report mind-wandering only if there is a natural break in their immersion, which we ensured them is entirely normal and acceptable. We used on established and extensively validated protocol [6]. After listening, participants were asked to fill out a postexperience questionnaire and return to Zoom for a follow-up discussion. During the discussion, participants were prompted by the moderator to reflect on their experiences with the protocol-namely, whether and how the presence of others and the request to report mind-wandering [21] affected their experience.

The primary focus of the research was on developing measures of audience engagement based on computer-vision analysis of non-verbal behaviour. These analyses are ongoing but once developed, such indexes could be integrated into performances, both as an overall evaluation and a way to enable audience-performance interactions in remote settings (e.g., alert a performer that the audience is engaged). Two insights revealed through content analysis of the audience experience discussions were that: a) the presence of others was enjoyable but the full-fledged videosoverbearing; b) reporting momentary disengagement did not feel forced or distracting. The latter finding was corroborated by quantitative analysis showing no significant differences in post-experience evaluations for sessions with and without momentary reports. Multiple participants expressed that they would have enjoyed seeing how other people react, for example, whether they danced to the music, but would have found it overwhelming to continuously see all participants' videos. When this concern was raised, the moderator followed-up with a description of a possible interface, where others are visible but less distinct, for example low brightness, over-imposed masking, or abstract representation such as point light display. These ideas were largely met with enthusiasm.

\subsection{Ergo Sum Multi-Sensory Theatre Production}

Ergo Sum is a multi-sensory theatre production which was due to be performed at Bristol Old Vic during summer 2020 and was postponed due to the pandemic. Ellie Chadwick, with theatre company Sleight of Hand Theatre, is redeveloping elements of the original show into an online multi-sensory storytelling experience using StoryFormer. Through a combination of video, binaural sound, text and interactive elements, audience members will navigate the world of three characters with mental and neurological conditions. They will be able to make choices and answer questions throughout as part of the interaction with the narratives and share reactions via social media.

The research will consist of three phases: a) Initial basic data-gathering on effects of binaural sound immersion using a cloud-based service for online experiments (Gorilla [28]): visual analogue surveys, responding to a set of segments with different sound environments, measuring the effects of binaural sound immersion on a sense of connection, empathy, emotional engagement; 2) Public audience experience (via StoryFormer): a full narrative experience, episodic; 3) Video analysis study (via StoryFormer and Zoom): full narrative experience, video analysis of engagement. 
The staging of Ergo Sum is still in progress and no audience data has been gathered yet. Our recommendations are drawn on the basis of extensive desk research and conversations with industry and academia undertaken by theatre director Ellie Chadwick in preparation of adapting the Ergo Sum for remote testing and delivery. The need to rely on multiple tools and adapt content to their capabilities is an indication that a sufficiently comprehensive technology has not yet been developed. The research into available tools and their limitations fed into our recommendations.

\section{DESIGN RECOMMENDATIONS}

We propose that the design of digital experiences and supporting technology could be enhanced through: a) Allowing for subtle audience visualisation and interaction, to enhance togetherness without breaking immersion; b) Integrating audience feedback and responses into experiences, to draw effective audience insights; c) Creating a sense of place, to enhance immersion and togetherness; d) Ensuring good ethics and inclusivity by design.

Allowing for subtle audience visualisation and interaction, to enhance togetherness

Shared experiences, or "togetherness" (audience-audience and performance-audience interactions) are a key element of live performances [15]. One challenge in the streaming of arts is the tension between sensory immersion and interactivity [18]. Traditional venues seamlessly afford conversations in lobbies and queues, ambient interaction in the form of coughing, clapping, fidgeting. Their digital counterparts, such as comments and participant videos, however, can be overpowering and detract from the primary content. It is important to consider such unintended psychological effects and how to deliver audience representation, which blends into performances without breaking immersion. One example would be reducing the amount of information to key elements. Some research suggests that when it comes to social presence, behavioural fidelity or realism, that is, the extent to which avatars and other objects in a virtual environment behave like their correspondents in the physical world [2], might be more important than visual fidelity. In a recent study [9], participants embodied and interacted with virtual avatars who tracked their actual head and hand movements; Participants whose virtual avatars consisted only of floating heads and hands experienced a greater connection than participants with full-body avatars, where only hands and head corresponded to real motion. Another paper reports increased empathy for a stigmatized group when minimal information about bio-signals (heart rate) was displayed alongside text in an interview transcript [12]. These findings are in line with psychological and biological insights into social perception-namely that abstract representations can drive emotional and behavioural responses.

An interesting point of comparison in the field of performance practice is provided by immersive theatre company Punchdrunk, at their shows audience members are required to wear masks while being allowed to freely wander in the space. These masks enhance the cohesive feeling of being a part of a wider collective of audience members, while individual experience is simultaneously enhanced by giving a level of anonymity that encourages personal freedom to explore the immersive world [11]. This individual freedom is always there yet is also "always giving way to the sense that one is very much part of a group and of the larger entity of the performance itself" [11]. In terms of creating a digital experience with elements of social cohesion, individual immersivity and interactivity, it is interesting to consider how deindividuation via avatars, symbols or other non-specific representations can be effectively utilised in audience representation; "deindividuating settings do not lead to a loss of personal identity; rather, they can facilitate a transition from a personal to a more social or collective identity, with its own highly contextual norms of behaviour" [14].

To create a sense of shared experience or "togetherness" whilst respecting privacy and avoiding perceptual overload, media players can incorporate abstract audience visualisations, which are subtly interactive. Making social features subtle would also accommodate different preferences for interaction and agency-people who prefer solitary experiences should be able to hide or easily ignore these elements. Finding an optimal means of visualising audiences and empirically testing the added value of such visualisations are questions for future research. There is a rich body of work on ambient visualisation in human-computer interaction research, which can help inform the design of effective visualisations for performance arts. 


\subsection{Integrating audience feedback and responses into experiences}

Audience research plays a crucial role in the creative industry and finding effective ways to conduct it is essential. Critically, some of the most powerful and reliable metrics of audience experience and engagement are missing from current platforms and practices. Asking people about cognitive and emotional experiences, such as immersion, is not necessarily reliable. Self-report measures reflect people's recollections of or narrative around experiences, which are known to be biased and dependent on unreliable human memory [13]. While not unimportant, such measures cannot tell the full story, as behaviour is often driven by the actual experience, rather than these post-factual rationalisations. Momentary experiences can be measured indirectly, through the analysis of physiology and non-verbal behaviour [23]. These approaches to audience research are novel and currently being developed, our own research around immersion being one example, but they will likely become widely used in the future. The time is ripe to begin thinking about how to best incorporate them in performances and the infrastructure supporting them.

Audience research can be incorporated into the design of streaming platforms, in ethical and engaging ways. Platforms can collect behavioural data such as user trajectories and detailed viewer statistics; the option to capture video recordings of users as they experience content; momentary self-report in the form of reactions (clapping, smiling) and embedded polls. Audience discussions can be moderated using participatory qualitative research software [17], even made playful through chatbots designed to provoke reflection [19].

Merely embedding research elements within experiences does not guarantee participant engagement; How to make research engaging and meaningful requires further research. Among the key needs identified in Radbourne et al. [2] seminal work on audience experience, were agency-or the need to have some impact on the environment or performance-and sense-making-the opportunity to reflect on experiences. The needs for agency and reflection can motivate research participation. Providing momentary feedback which feeds into the performance or a shared visualization, is a form of agency. Post-experience questionnaires and discussions allow audiences to reflect on their experiences and can be designed to support personal insights, for example, by providing background information.

\subsection{Creating a sense of place}

Physical spaces and the critical role they play in audience experience can be reimagined for digital platforms. Traditional venues are designed to provide optimal conditions in which to experience content: to enhance immersion, emphasis is placed on content, distractions are reduced or discouraged; they provide a separation between everyday life and the fictional world of performances, which may be conducive to immersion [16]; transition points, or liminal spaces, between performances and everyday life, in which people can connect and reflect $[\underline{2}, \underline{6}]$.

In all three of our studies, it became apparent that physical locations provide a natural platform for some key elements of audience experience and research, that their existing digital counterparts do not. Before a performance, theatre lobbies provide an opportunity for audiences to break away from their usual environment, as well as to gather and form a sense of shared identity or togetherness. Afterwards, they can be used to gather feedback and gauge audiences into discussions. Since the beginning of the pandemic, platforms have emerged that begin to address this problem-the event-hosting platform Hopin is a notable example. Activities on Hopin take place in virtual rooms, which users access from a main page, akin to a lobby. Participants can interact outside of the main event through chat or video. Such a setup can provide room for audience interactions and reflection, much like a theatre lobby. Importantly, creators can be given control over how these spaces tie into a performance. With appropriate informed consent, audience behaviour and discussions can be recorded and used in audience research.

\subsection{Ensuring good ethics and inclusivity by design}

Technology can and should be made as inclusive as possible. This includes basic accessibility, such as captioning content; ensuring good core functionality does not depend upon high-end technical resources. When developing experiences and platforms on the basis of existing research, it should be acknowledged that published research often relies on convenience sampling and is not sufficiently diverse [8]. Computer-vision algorithms have been shown to underperform on samples different to the ones used to develop and validate them [5]. In terms of audience 
engagement, there has been a debate as to whether conventional indicators of engagement, such as stillness, are not generalizable across cultures, demographics, and neurodiversity [20].

When incorporating emotion recognition in design it is crucial to consider users' attitudes and concerns, as such technology tends to be perceived by users as "intimate, vulnerable, and complex" [1] . Any collection of personal data, especially sensitive data such as video recordings, needs to be held to high ethical standards-a responsibility which begins in the design and development of platforms. In addition to impeccable cybersecurity, platforms can incorporate unambiguous consent forms to ensure audience members are fully informed about the research they are being involved in and the data being collected. Functions involving the collection of sensitive, personally-identifiable data can be restricted and made available only to users with formal ethical clearance.

\section{DISCUSSION}

In this paper we share design recommendations for the digital delivery of performance arts, which we drew from a set of early-stage work with audiences during the COVID19 pandemic. Guided by the question of how technology can be used to support the needs of artists and audiences, we focused on audiences' needs for immersion, togetherness, and reflection, as well as the need of content producers to gain insight into audiences' experiences and behaviour. We formulated the following recommendations:

- Allowing for subtle audience visualisation and interaction, to enhance togetherness

- Integrating audience feedback and responses into experiences, to draw audience insights

- Creating a sense of place in digital settings, to enhance immersion and togetherness

- Ensuring ethical conduct and inclusivity

The research on which these recommendations are based was conducted within a research and development collaboration between universities and the creative industry, focused on Expanded Performance-exploring how emerging technology can be used in performance arts. This unique and timely collaboration, allowed us to approach the problem of digital delivery of performance arts from multiple perspectives, including social and cognitive psychology, computer science, theatre studies, and the creative industry.

One limitation of this paper is that the insights we share emerged in the margins of work with separate aims, where experience design was not the main focus. These methodological limitations notwithstanding, the ideas we put forward are grounded in applied work and a diverse set of relevant perspectives and constitute a valuable contribution to the fields of performance arts and human-computer interaction. Follow-up research with appropriate user experience methodology and relevant stakeholders will be conducted to refine and improve our recommendations.

Our paper puts forward some design recommendations on how to enhance remote experiences of performing arts, based on pilot conducted during the COVID19 pandemic. The focus was on how technology can be used to enhance key audience experiences of immersion, togetherness, and reflection, along with effective audience research. The topic is extremely timely given the ongoing restrictions imposed by the pandemic. Importantly, the implications of our work extend beyond this immediate situation-remote experiences are the permanent reality for many individuals who cannot attend performances in person, for various reasons, including disabilities, financial or time constraints. Furthermore, the core audience needs around which our work was based-immersion, togetherness, reflection-extend to human-centred design beyond the domain of performing arts

\section{Acknowledgements}

We would like to thank the Bristol Arts Channel organisers and Bristol and Bath Creative R\&D team and fellows for numerous inspiring conversations This work was supported by Bristol and Bath Creative R\&D funded by the AHRC Creative Industries Cluster Programme. For more information on the project and its partners, visit our website at https://bristolbathcreative.org/

\section{References}

[1] Nazanin Andalibi and Justin Buss. 2020. The Human in Emotion Recognition on Social Media: Attitudes, Outcomes, Risks. Conference on Human Factors in Computing Systems - Proceedings: 1-16.

[2] BBC. StoryFormer. Retrieved from https://www.bbc.co.uk/makerbox/tools/storyformer

[3] Bristol and Bath Creative R\&D. Expanded Performance Pathfinder. Retrieved from https://bristolbathcreative.org/pathfinders/expandedperformance.

[4] Alan S Brown and Rebecca Ratzkin. 2011. Making Sense of Audience Engagement. The San Francisco Fundation. 
[5] Cynthia M. Cook, John J. Howard, Yevgeniy B. Sirotin, Jerry L. Tipton, and Arun R. Vemury. 2019. Demographic Effects in Facial Recognition and Their Dependence on Image Acquisition: An Evaluation of Eleven Commercial Systems. IEEE Transactions on Biometrics, Behavior, and Identity Science.

[6] Myrthe Faber, Robert Bixler, and Sidney K. D’Mello. 2018. An automated behavioral measure of mind wandering during computerized reading. Behavior Research Methods 50, 1: 134-150.

[7] Melanie C. Green, Timothy C. Brock, and Geoff F. Kaufman. 2004. Understanding media enjoyment: The role of transportation into narrative worlds. Communication Theory.

[8] Joseph Henrich, Steven J. Heine, and Ara Norenzayan. 2010. The weirdest people in the world? Behavioral and Brain Sciences.

[9] Fernanda Herrera, Soo Youn Oh, and Jeremy N. Bailenson. 2020. Effect of behavioral realism on social interactions inside collaborative virtual environments. Presence: Teleoperators and Virtual Environments.

[10] Katherine Jewkes and Clare Reddington. 2020. Bristol Arts Channel: Pilot Season Summary Report. .

[11] Myrto Koumarianos and Cassandra Silver. 2013. Dashing at a nightmare: Haunting Macbeth in sleep no more. TDR - The Drama Review - A fournal of Performance Studies.

[12] Fannie Liu, Laura Dabbish, and Geoff Kaufman. 2017. Supporting Social Interactions with an Expressive Heart Rate Sharing Application. Proceedings of the ACM on Interactive, Mobile, Wearable and Ubiquitous Technologies.

[13] Talya Miron-Shatz, Arthur Stone, and Daniel Kahneman. 2009. Memories of Yesterday's Emotions: Does the Valence of Experience Affect the Memory-Experience Gap? Emotion

[14] Tom Postmes and Russell Spears. 1998. Deindividuation and Antinormative Behavior: A Meta-Analysis. Psychological Bulletin.

[15] Jennifer Radbourne, Katya Johanson, Hilary Glow, and Tabitha White. 2009. Audience experience: measuring quality in the performing arts. International journal of arts management.

[16] Gabriel A. Radvansky and David E. Copeland. 2006. Walking through doorways causes forgetting: Situation models and experienced space. Memory and Cognition.

[17] Jay Rainey, Kyle Montague, Pamela Briggs, Robert Anderson, Thomas Nappey, and Patrick Olivier. 2019. GabbEr: Supporting voice in participatory qualitative practices. Conference on Human Factors in Computing Systems - Proceedings.

[18] Asreen Rostami, Chiara Rossitto, and Annika Waern. 2018. Frictional realities: Enabling immersion in mixed-reality performances. TVX 2018 Proceedings of the 2018 ACM International Conference on Interactive Experiences for TV and Online Video.

[19] Maria Roussou, Sara Perry, Akrivi Katifori, Stavros Vassos, Angeliki Tzouganatou, and Sierra McKinney. 2019. Transformation through provocation? Designing a 'bot of conviction' to challenge conceptions and evoke critical reflection. Conference on Human Factors in Computing Systems - Proceedings: $1-13$.

[20] Kirsty Sedgman. 2018. The reasonable audience: Theatre etiquette, behaviour policing, and the live performance experience.

[21] Jonathan Smallwood and Jonathan W. Schooler. 2006. The restless mind. Psychological Bulletin 132, 6: 946-958.

[22] K Swinth and Jim Blascovich. 2002. Perceiving and responding to others: Human-human and human-computer social interaction in collaborative virtual environments. ISPR Presence conference 2002.

[23] Lida Theodorou, Patrick G.T. Healey, and Fabrizio Smeraldi. 2019. Engaging with contemporary dance: What can body movements tell us about audience responses? Frontiers in Psychology 10, FEB: 1-13.

24] Andreas Wagener. 2012. Why Do People (Not) Cough in Concerts? The Economics of Concert Etiquette. ACEI working paper series December.

[25] Chen Wang, Erik Geelhoed, Phil Stenton, and Pablo Cesar. 2014. Sensing a live audience. Conference on Human Factors in Computing Systems Proceedings: 1909-1912.

[26] Hopin: The first all-in-one live online events platform. Retrieved from https://hopin.com/.

[27] YouTube Live. Retrieved from https://www.youtube.com/live/all.

[28] Gorilla: Experiment Builder. Retrieved from https://gorilla.sc/. 